\title{
An Atypical Case of Brugada Syndrome
}

\author{
Almpanis Georgios, M.D., Servos Georgios, M.D., Tragotsalou Nikolitsa, M.D., \\ Koutsogiannou Persefoni, M.D., and Mazarakis Andreas, M.D. \\ From the "Agios Andreas" General Hospital of Patras, 1st Cardiology Department, Tsertidou 1, Patra, Greece
}

\begin{abstract}
Polymorphic ventricular tachycardia and ventricular fibrillation are the most common arrhythmias in Brugada syndrome causing syncope or sudden death. Sustained monomorphic ventricular tachycardias are rare in this context. We report of a patient with syncopal episodes due to episodes of sustained ventricular tachycardia, where a Type-I Brugada pattern was revealed after pharmacological provocation with procainamide.
\end{abstract}

Ann Noninvasive Electrocardiol 2011;16(4):412-414

Brugada syndrome; electrocardiography; ventricular tachycardia/fibrillation

\section{CASE REPORT}

A 57-year-old man presented at the emergency room (ER) because of syncope with concurrent facial head injury. In the ER, he experienced a second episode (further injury was prevented, as he was promptly put in supine position). Immediately after the episode he had an electrocardiogram (ECG) (Fig. 1). Tachycardia resolved without any medical intervention in not more than 30 seconds. There was no history of previous syncope, no personal history of coronary artery disease, and no familial history of sudden cardiac death (SCD). His physical examination and laboratory tests were unremarkable. He was transferred to the coronary care unit (CCU), where while being monitored had another episode of monomorphic ventricular tachycardia at 230 beats per minute with the same morphology, minimally symptomatic while lying in bed. He was converted to sinus rhythm with I.V. amiodarone at $15 \mathrm{mg} / \mathrm{min}$. A loading dose of I.V. amiodarone was started and low dose of I.V. esmolol was added, regarding his borderline sinus heart rate and the PR interval of $238 \mathrm{msec}$. The patient remained stable without symptoms or recurrent episodes for 24 hours. cTnI was negative in three consecutive measurements during the first 24 hours. The patient had a bedside echocardiogram that was interpreted as within normal limits for his age. The following (Fig. 2) is the ECG recorded next morning in the CCU. This was characterized as Type-III
Brugada ECG. Amiodarone loading was stopped and the patient underwent a provocation test with procainamide, a $10 \mathrm{mg} / \mathrm{kg}$ over $10 \mathrm{~min}$ infusion which revealed a coved-type (Type-I) Brugada ECG pattern, in $\mathrm{V}_{1}-\mathrm{V}_{2}$ leads (Fig. 3). His coronary angiogram plus left and right ventriculography were within normal limits. The patient was referred to the regional electrophysiologic laboratory where an implantable cardioverter-defibrillator (ICD) was implanted.

\section{DISCUSSION}

Brugada syndrome was described as entity causing unexpected SCD in the absence of cardiac abnormalities. ${ }^{1}$ Three patterns of depolarization have been described, but only Type-1 morphology is diagnostic, either spontaneous or provoked by potent sodium channel blockers. ${ }^{2}$

SCN5A mutations, found at $18-30 \%$ of subjects with Brugada syndrome, are often associated with other arrhythmic entities such as long QT syndrome and atrioventricular block. ${ }^{3}$ In Brugada syndrome, SCN5A mutations, causing dysfunction of a-subunit of the sodium channel, result in loss of function due to a plethora of mechanisms. ${ }^{4}$ The remaining 70-75\% subjects do not necessarily have other genes mutations as negative SCN5A screening does not rule out mutations at the promoter region of the gene, cryptic splicing mutations, or presence of gross rearrangements. ${ }^{4}$ Other 


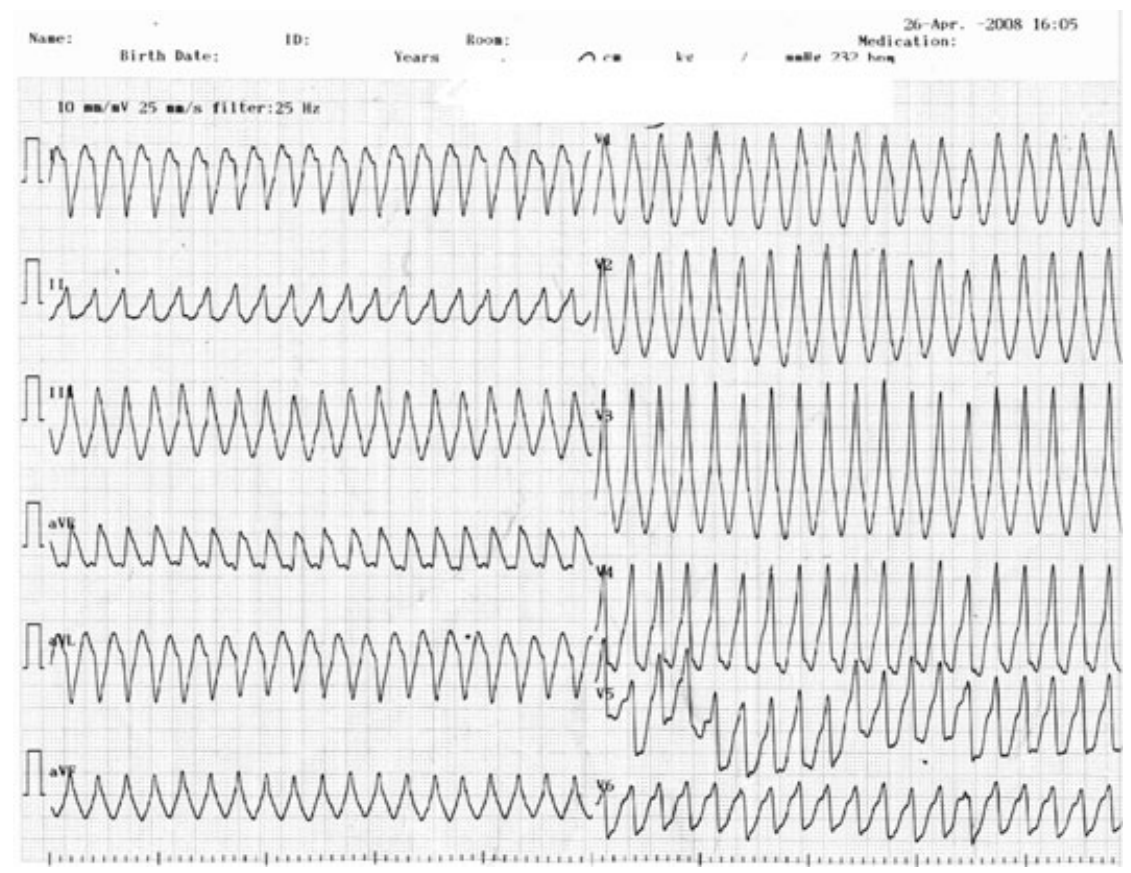

Figure 1. Twelve-lead ECG showing monomorphic VT.

mutations found to be linked to the syndrome ${ }^{5}$ cause a more benign phenotype. ${ }^{6}$

Polymorphic ventricular tachycardia and ventricular fibrillation are the only arrhythmias included in the definition of Brugada syndrome. ${ }^{2}$
They are postulated to be a circus movement tachycardia initiated by an early coupled premature ventricular complex following a phase-2 reentry due to a dispersion of repolarization in right ventricular epicardium. Sustained monomorphic

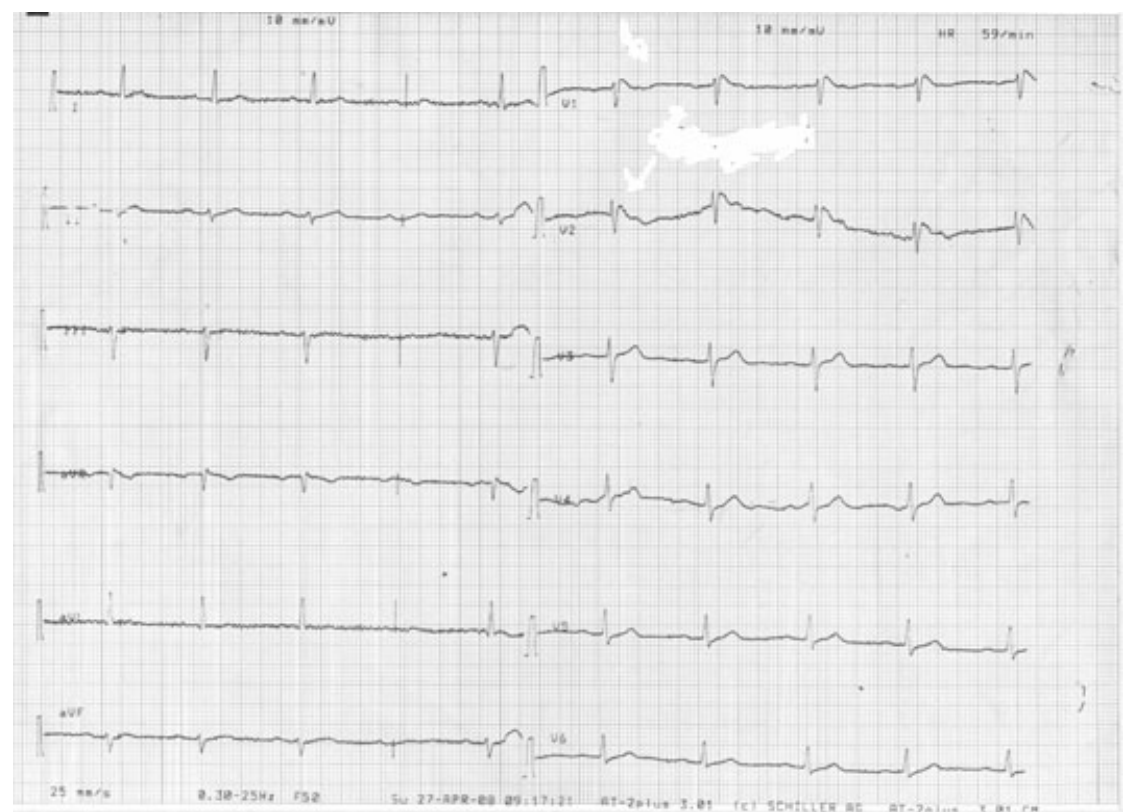

Figure 2. Two-lead ECG showing a Type-3 Brugada pattern, raised the suspicion for Brugada syndrome. 


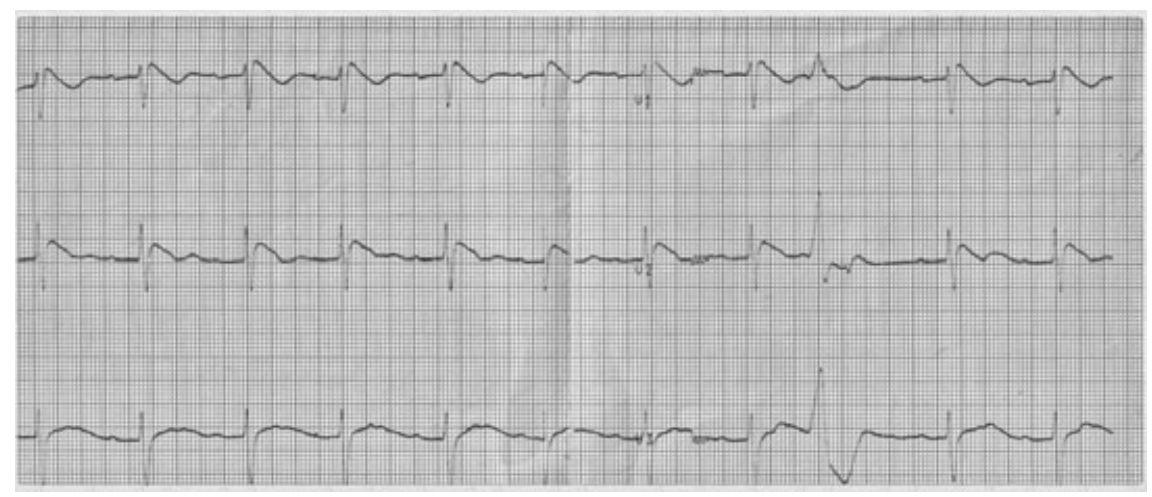

Figure 3. A Type-1 Brugada pattern appears after procainamide administration.

ventricular tachycardias are rare in this context but they have been described. ${ }^{7}$ The mechanism underlying sustained morphometric ventricular tachycardia in Brugada syndrome remains unknown. Both triggered automaticity and phase- 2 reentry ${ }^{8}$ have been suggested.

Our patient suffered a syncopal episode, caused by monomorphic ventricular tachycardia, with right bundle branch block morphology and right axis deviation. This kind of tachycardia usually originates from LV in the setting of ischemic heart disease. But our patient had an unremarkable baseline ECG, negative troponine, normal echo, coronary angiography, and left and right ventriculography, mostly excluding structural heart disease. Administration of intravenous amiodarone unmasked Type-3 morphology in the right precordial leads probably due to its sodium blocker properties, which are enhanced by rapid I.V. infusion, ${ }^{9}$ and that is why administration of amiodarone should be avoided in Brugada patients. The ECG following procainamide provocation was beyond doubt diagnostic. Even though the SCN5A defect is immediately linked to electrical phenomena and late potentials related to the RVOT, the presence of this channel is well documented to be widespread in various myocardial areas. Electrophysiological disturbances such as prolonged sinus atrial recovery time and sinoatrial conduction time, PR and HV interval prolongation, even a slight prolongation of the QT interval, has been described in this setting. This multitude of electrophysiologic disturbances pose a possible explanation for the development of monomorphic ventricular tachycardia, causing conduction abnormality possible associated with reentry, ${ }^{10}$ in the anterosuperior segment of $L V$, in our case.
EP study was not conducted so we treated the patient by ICD device, as we believed he was at an increased risk of SCD. We closely follow the patient throughout the last year, without evidence of untoward recurrent events.

\section{REFERENCES}

1. Brugada P, Brugada J. Right bundle branch block, persistent ST segment elevation and sudden cardiac death: A distinct clinical and electrocardiographic syndrome. A multicenter report. J Am Coll Cardiol 1992;20:1391-1396.

2. Antzelevitch C, Brugada P, Borggrefe M, et al. Brugada syndrome: Report of the second consensus conference: Endorsed by the Heart Rhythm Society and the European Heart Rhythm Association. Circulation 2005;111:659-670.

3. Wang DW, Viswanathan PC, Balser JR, et al. Clinical, genetic, and biophysical characterization of SCN5A mutations associated with atrioventricular conduction block. Circulation 2002;105:341-346.

4. Antzelevitch C. Genetic basis of Brugada syndrome. Heart Rhythm 2007;4:756-757.

5. Weiss $R$, Barmada MM, Nguyen $T$, et al. Clinical and molecular heterogeneity in the Brugada syndrome: A novel gene locus on chromosome 3. Circulation 2002;105: 707-713.

6. Pfahnl AE, Viswanathan PC, Weiss R, et al. A sodium channel pore mutation causing Brugada syndrome. Heart Rhythm 2007;4:46-53.

7. Allocca G, Proclemer A, Nucifora G, et al. Monomorphic ventricular tachycardia in 'Brugada syndrome': Clinical case and literature review. J Cardiovasc Med (Hagerstown) 2008;9:842-856.

8. Pinar Bermudez E, Garcia-Alberola A, Martinez Sanchez J, et al. Spontaneous sustained monomorphic ventricular tachycardia after administration of ajmaline in a patient with Brugada syndrome. Pacing Clin Electrophysiol 2000;23: 407-409.

9. Mitchell LB, Wyse DG, Gillis AM, et al. Electropharmacology of amiodarone therapy initiation. Time courses of onset of electrophysiologic and antiarrhythmic effects. Circulation 1989;80:34-42.

10. Yan GX, Antzelevitch C. Cellular basis for the Brugada syndrome and other mechanisms of arrhythmogenesis associated with ST-segment elevation. Circulation 1999;100: 1660-1666. 